\title{
The Linear Span of Projections in AH Algebras and for Inclusions of $C^{*}$-Algebras
}

\author{
Dinh Trung Hoa, ${ }^{1}$ Toan Minh Ho, ${ }^{2}$ and Hiroyuki Osaka ${ }^{3}$ \\ ${ }^{1}$ Center of Research and Development, Duy Tan University, K7/25 Quang Trung, Da Nang, Vietnam \\ ${ }^{2}$ Institute of Mathematics, VAST, 18 Hoang Quoc Viet, Ha Noi 10307, Vietnam \\ ${ }^{3}$ Department of Mathematical Sciences, Ritsumeikan University, Kusatsu, Shiga 525-8577, Japan
}

Correspondence should be addressed to Hiroyuki Osaka; osaka@se.ritsumei.ac.jp

Received 18 October 2012; Accepted 6 January 2013

Academic Editor: Ivanka Stamova

Copyright (C) 2013 Dinh Trung Hoa et al. This is an open access article distributed under the Creative Commons Attribution License, which permits unrestricted use, distribution, and reproduction in any medium, provided the original work is properly cited.

In the first part of this paper, we show that an AH algebra $A=\lim \left(A_{i}, \phi_{i}\right)$ has the LP property if and only if every element of the centre of $A_{i}$ belongs to the closure of the linear span of projections in $A$. As a consequence, a diagonal AH-algebra has the LP property if it has small eigenvalue variation in the sense of Bratteli and Elliott. The second contribution of this paper is that for an inclusion of unital $C^{*}$-algebras $P \subset A$ with a finite Watatani index, if a faithful conditional expectation $E: A \rightarrow P$ has the Rokhlin property in the sense of Kodaka et al., then $P$ has the LP property under the condition that $A$ has the LP property. As an application, let $A$ be a simple unital $C^{*}$-algebra with the LP property, $\alpha$ an action of a finite group $G$ onto Aut $(A)$. If $\alpha$ has the Rokhlin property in the sense of Izumi, then the fixed point algebra $A^{G}$ and the crossed product algebra $A \rtimes_{\alpha} G$ have the LP property. We also point out that there is a symmetry on the CAR algebra such that its fixed point algebra does not have the LP property.

\section{Introduction}

A $C^{*}$-algebra is said to have the LP property if the linear span of projections (i.e., the set of all linear combinations of projections in the algebra) is dense in this algebra. A picture of the problem which asks to characterize the simple $C^{*}$ algebras to have the LP property was considered in [1]. The LP property of a $C^{*}$-algebra is weaker than real rank zero since the latter means that every self-adjoint element can be arbitrarily closely approximated by linear combinations of orthogonal projections in this $C^{*}$-algebra. In the class of simple AH algebras with slow dimension growth, real rank zero and small eigenvalue variation in the sense of Bratteli and Elliott are equivalent (see $[2,3])$. It is not known whether the equivalence still holds when the algebras do not have slow dimension growth.

The concept of diagonal AH algebras (AH algebra which can be written as an inductive limit of homogeneous $C^{*}$ algebras with diagonal connecting maps) was introduced in [4] or [5]. Let us denote by $\mathscr{D}$ the class of diagonal AH algebras. AF-, AI-, and AT-algebras, Goodearl algebras [6], and Villadsen algebras of the first type [7] are diagonal AH algebras. The algebras constructed by Toms in [8] specially which have the same $K$-groups and tracial data but different Cuntz semigroups are Villadsen algebras of the first type and so belong to $\mathscr{D}$. This means that the class $\mathscr{D}$ contains "ugly" and interesting $C^{*}$-algebras and has not been classified by Elliott's program so far.

Note that the classification program of Elliott, the goal of which is to classify amenable $C^{*}$-algebras by their $K$ theoretical data, has been successful for many classes of $C^{*}$ algebras, in particular for simple AH algebras with slow dimension growth (see, e.g., [9-11]). Unfortunately, for AH algebras with higher dimension growth, very little is known.

In the first part of this paper (Section 2), we consider the LP property of inductive limits of matrix algebras over $C^{*}$ algebras. The necessary and sufficient conditions for such an inductive limit to have the LP property will be presented in Theorem 1. In particular, we will show that an $\mathrm{AH}$ algebra $A=\lim \left(A_{i}, \phi_{i}\right)$ (which need not be diagonal nor simple) 
has the LP property provided that the image of every element of the centre of the building blocks $A_{i}$ can be approximated by a linear combination of projections in $A$ (Corollary 2). In Section 2.4, using the idea of bubble sort, we can rearrange the entries on a diagonal element in $M_{n}(C(X))$ to obtain a new diagonal element with increasing entries such that the eigenvalue variations are the same (Lemma 5) and the eigenvalue variation of the latter element is easy to evaluate. As a consequence, it will be shown that a diagonal $\mathrm{AH}$ algebra has the LP property if it has the small eigenvalue property (Theorem 6) without any condition on the dimension growth.

It is well known that the LP property of a $C^{*}$-algebra $A$ is inherited to the matrix tensor product $M_{n}(A)$ and the quotient $\pi(A)$ for any $*$-homomorphism $\pi$. But it is not stable under the hereditary subalgebra of $A$. In the second part of this paper (Section 3), we will present the stability of the LP property of an inclusion of a unital $C^{*}$-algebra with certain conditions and some examples illustrated the instability of such the property. More precisely, let $1 \in P \subset A$ be an inclusion of unital $C^{*}$-algebras with a finite Watatani index and $E: A \rightarrow P$ a faithful conditional expectation. Then the LP property of $P$ can be inherited from that of $A$ provided that $E$ has the Rokhlin property in the sense of Osaka and Teruya (Theorem 23). As a consequence, given a simple unital $C^{*}$ algebra $A$ with the LP property if an action $\alpha$ of a finite group $G$ to $\operatorname{Aut}(A)$ has the Rokhlin property in the sense of Izumi, then the fixed point algebra $A^{G}$ and the crossed product $A \rtimes_{\alpha} G$ have the LP property (Theorem 24). Furthermore, we also give an example of a simple unital $C^{*}$-algebra with the LP property, but its fixed point algebra does not have the LP property (Example 14).

Let us recall some notations. Throughout the paper, $M_{n}$ stands for the algebra of all $n \times n$ complex matrices, $e=$ $\left\{e_{s t}\right\}_{s, t=\overline{1, n}}$ denotes the standard basis of $M_{n}$ (for convenience, we also use this system of matrix unit for any size of matrix algebras). Let us denote by $M_{n}(C(X))$ the matrix algebra with entries from the algebra $C(X)$ of all continuous functions on space $X$. If $X$ has finitely many connected components $X_{i}$ and $X=\sqcup_{i=1}^{k} X_{i}$, then

$$
M_{n}(C(X))=\bigoplus_{i=1}^{k} M_{n}\left(C\left(X_{i}\right)\right)
$$

Hence, without lots of generality we can always assume that the spectrum of each component of a homogeneous $C^{*}$ algebra is connected.

Denote by $\operatorname{diag}\left(a_{1}, a_{2}, \ldots, a_{n}\right)$ the block diagonal matrix with entries $a_{1}, a_{2}, \ldots, a_{n}$ in some algebras.

Let $A$ be a $C^{*}$-algebra. Any element $a$ in $A$ can be considered as an element in $M_{n}(A)$ via the embedding $a \rightarrow$ $\operatorname{diag}(a, 0)$. We also denote by $L(A)$ the closure of the set of all linear combinations of finitely many projections in $A$.

The last two authors appreciate Duy Tan University for the warm hospitality during our visit in September 2012 and the third author would also like to thank Teruya Tamotsu for fruitful discussions about the $C^{*}$-index theory.

\section{Linear Span of Projections in AH Algebras}

2.1. Linear Span of Projections in an Inductive Limit of Matrix Algebras over $C^{*}$-Algebras. Let

$$
A=\lim _{\longrightarrow}\left(A_{i}, \varphi_{i}\right),
$$

where $A_{i}=\oplus_{t=1}^{k_{i}} M_{n_{i t}}\left(B_{i t}\right)$ and $B_{i t}$ are $C^{*}$-algebras. Let $S_{i t}$ be a spanning set of $B_{i t}$ (as a vector space) and $S_{i}$ be the union of $S_{i t}$ for $t=1, \ldots, k_{i}$. Since every element of a $C^{*}$-algebra can be written as a sum of two self-adjoint elements, we can assume that all elements of $S_{i}$ are self-adjoint.

Theorem 1. Let $A$ be an inductive limit $C^{*}$-algebra as above. Then the following statements are equivalent.

(i) A has the LP property.

(ii) For any integer $i$, any $x \in S_{i}$ and any $\varepsilon>0$, there exists an integer $j \geq i$ such that $\varphi_{i j}(x)$ can be approximated by an element in $L\left(A_{j}\right)$ to within $\varepsilon$.

(iii) For any integer $i$, there exist a spanning set of $A_{i}$ such that the images of all elements in that spanning set under $\varphi_{i \infty}$ belong to $L(A)$.

Proof. The implication (iii) $\Rightarrow$ (i) is obvious.

To prove the implication (i) $\Rightarrow$ (ii), it suffices to mention that every element (projection) in $A$ can be arbitrarily closely approximated by elements (projections, resp.) in $A_{i}$.

Let us prove the implication (ii) $\Rightarrow$ (iii). Clearly, without lots of generality we can assume that $A_{i}=M_{n_{i}}\left(B_{i}\right)$ for every $i$. For a fixed integer $i$, we put

$$
e \otimes S_{i}=\left\{e_{s t} \otimes x+e_{t s} \otimes x^{*}, x \in S_{i}\right\} .
$$

Hence, there exists a unitary $u \in M_{n_{i}}$ such that

$$
u\left(e_{s t} \otimes x+e_{t s} \otimes x\right) u^{*}=\left(\begin{array}{ccc}
0 & x & 0 \\
x & 0 & 0 \\
0 & 0 & 0
\end{array}\right),
$$

where the 0 in the last column and the last row is of order $n_{i}-2$.

It is evident that every element in $A_{i}$ is a linear combination of elements in $e \otimes S_{i} \cup D$, where $D$ is the set of all diagonal elements with coefficients in $S_{i}$. Thus, $e \otimes S_{i} \cup D$ is the spanning set of $A_{i}$. Now, we claim that this spanning set satisfies the requirement of (iii).

Firstly, let $d=\operatorname{diag}\left(x_{1}, \ldots, x_{n_{i}}\right)\left(x_{t} \in S_{i}\right)$ be an element in $D$. By (ii), $\varphi_{i \infty}\left(x_{t}\right) \in L(A)$ for every $t$. Hence $\varphi_{i \infty}(d) \in L(A)$. Lastly, let $a \in e \otimes S_{i}$. By Identity (4), $a$ can be assumed to be

$$
\left(\begin{array}{lll}
0 & x & 0 \\
x & 0 & 0 \\
0 & 0 & 0
\end{array}\right) \text { for some } x \in S_{i} \text {. }
$$

Moreover,

$$
a=u^{*}\left(\begin{array}{ccc}
-x & 0 & 0 \\
0 & x & 0 \\
0 & 0 & 0
\end{array}\right) u, \quad \text { where } u=\left(\begin{array}{ccc}
\frac{1}{\sqrt{2}} & \frac{1}{\sqrt{2}} & 0 \\
-\frac{1}{\sqrt{2}} & \frac{1}{\sqrt{2}} & 0 \\
0 & 0 & 1
\end{array}\right) \text {. }
$$


In addition, there exists an integer $j \geq i$ such that $\varphi_{i j}(x)$ can be approximated by an element of $L\left(A_{j}\right)$ to within $\mathcal{\varepsilon}$. Hence $\varphi_{i j}(a)$ can be approximated by an element of $L\left(A_{j}\right)$ to within $\varepsilon$.

Corollary 2. Let $A=\underset{\lim }{\longrightarrow}\left(A_{i}, \varphi_{i}\right)$ be an $A H$ algebra, where $A_{i}=\oplus_{t=1}^{k_{i}} M_{n_{i t}}\left(C\left(X_{i t}\right)\right)$ and $X_{i t}$ are connected compact Hausdorff spaces. Then the following statements are equivalent.

(i) A has the LP property.

(ii) For any integer $i$, any $f \in \cup_{t=1}^{k_{i}} C\left(X_{i t}\right)$ and any $\varepsilon>0$, there exists an integer $j \geq i$ such that $\varphi_{i j}(f)$ can be approximated by an element in $L\left(A_{j}\right)$ to within $\varepsilon$.

From the proof of Theorem 1, we can obtain the following.

Corollary 3. Let $A$ be a $C^{*}$-algebra. If $A$ has the LP property, then $A \otimes M_{n}$ and $A \otimes K$ have the LP property, where $K$ is the algebra of compact operators on a separable Hilbert space.

2.2. Linear Span of Projections in a Diagonal AH Algebra. For convenience of the reader, let us recall the notions from [4]. Let $X$ and $Y$ be compact Hausdorff spaces. A *homomorphism $\phi$ from $M_{n}(C(X))$ to $M_{n m+k}(C(Y))$ is said to be diagonal if there exist continuous maps $\left\{\lambda_{i}, i=\overline{1, n}\right\}$ from $Y$ to $X$ such that

$$
\begin{array}{r}
\phi(f)=\operatorname{diag}\left(f \circ \lambda_{1}, f \circ \lambda_{2}, \ldots, f \circ \lambda_{n}, 0\right), \\
f \in M_{n}(C(X)),
\end{array}
$$

where 0 is a zero matrix of order $k(k \geq 0)$. If the size $k=0$, the map is unital.

The $\lambda_{i}$ are called the eigenvalue maps (or simply eigenvalues) of $\phi$. The family $\left\{\lambda_{1}, \lambda_{2}, \ldots, \lambda_{m}\right\}$ is called the eigenvalue pattern of $\phi$. In addition, let $p$ and $q$ be projections in $M_{n}(C(X))$ and $M_{n m+k}(C(Y))$, respectively. An *homomorphism $\psi$ from $p M_{n}(C(X)) p$ to $q M_{n m+k}(C(Y)) q$ is called diagonal if there exists a diagonal $*$-homomorphism $\phi$ from $M_{n}(C(X))$ to $M_{n m+k}(C(Y))$ such that $\psi$ is reduced from $\phi$ on $p M_{n}(C(X)) p$ and $\phi(p)=q$. This definition can also be extended to a $*$-homomorphism

$$
\phi: \bigoplus_{i=1}^{n} p_{i} M_{n_{i}}\left(C\left(X_{i}\right)\right) p_{i} \longrightarrow \bigoplus_{j=1}^{m} q_{j} M_{m_{j}}\left(C\left(Y_{j}\right)\right) q_{j}
$$

by requiring that each partial map

$$
\phi^{i, j}: p_{i} M_{n_{i}}\left(C\left(X_{i}\right)\right) p_{i} \longrightarrow q_{j} M_{m_{j}}\left(C\left(Y_{j}\right)\right) q_{j}
$$

induced by $\phi$ be diagonal.

2.3. Eigenvalue Variation. Suppose that $B$ is a simple $A H$ algebra. Then, $B$ has real rank zero if and only if its projections separate the traces provided that this algebra has slow dimension growth (see [12]). This equivalence was first studied when the dimensions of the spectra of the building blocks in the inductive limit decomposition of $B$ are not more than two, see [2].
Let $B$ be a $C^{*}$-algebra. Suppose that

$$
B=\bigoplus_{i=1}^{k} C\left(X_{i}\right) \otimes M_{n_{i}}
$$

where $X_{i}$ is a connected compact Hausdorff space for every $i$. Set $X=\sqcup_{i=1}^{k} X_{i}$. The following theorem and notations are quoted from $[2,12]$.

Let $a$ be any self-adjoint element in $B$. For any $x$ in $X_{i}$, any positive integer $m, 1 \leq m \leq n_{i}$, let $\lambda_{m}$ denote the $m$ th lowest eigenvalue of $a(x)$ counted with multiplicity. So $\lambda_{m}$ is a function on each $X_{i}$, for $i=1,2, \ldots, k$. The fact is

$$
\left|\lambda_{m}(x)-\lambda_{m}(y)\right| \leq\|a(x)-a(y)\| \text {. }
$$

Hence, $\lambda_{m}$ is continuous, for $m=1,2, \ldots, k$ for a given summand of $B$.

The variation of the eigenvalues of $a$, denoted by $\operatorname{EV}(a)$, is defined as the maximum of the nonnegative real numbers

$$
\sup \left\{\left|\lambda_{m}(x)-\lambda_{m}(y)\right| ; x, y \in X_{i}\right\}
$$

over all $i$ and all possible values of $m$.

The variation of the normalized trace of $a$, denoted by $\mathrm{TV}(a)$, is defined as

$$
\begin{array}{r}
\sup \left\{\left|\frac{1}{n_{i}} \sum_{m=1}^{n_{i}}\left(\lambda_{m}(x)-\lambda_{m}(y)\right)\right| ; x, y \in X_{i}\right\} \\
=\sup \left\{|\operatorname{tr}(a(x))-\operatorname{tr}(a(y))| x, y \in X_{i}\right\}
\end{array}
$$

over all $i$, where tr denotes the normalized trace of $M_{n}$ for any positive integer $n$.

Theorem 4 (see [2]). Let $B$ be an inductive limit of homogeneous $C^{*}$-algebras $B_{i}$ with morphisms $\phi_{i j}$ from $B_{i}$ to $B_{j}$. Suppose that $B_{i}$ has the form

$$
B_{i}=\bigoplus_{t=1}^{k_{i}} M_{n_{i t}}\left(C\left(X_{i t}\right)\right)
$$

where $k_{i}$ and $n_{i t}$ are positive integers, and $X_{i t}$ is a connected compact Hausdorff space for every positive integer $i$ and $1 \leq$ $t \leq k_{i}$. Consider the following conditions.

(1) The projections of $B$ separate the traces on $B$.

(2) For any self-adjoint element $a$ in $B_{i}$ and $\varepsilon>0$, there is a $j \geq i$ such that

$$
T V\left(\phi_{i j}(a)\right)<\varepsilon
$$

(3) For any self-adjoint element $a$ in $B_{i}$ and any positive number $\varepsilon$, there is a $j \geq i$ such that

$$
E V\left(\phi_{i j}(a)\right)<\varepsilon
$$


(4) B has real rank zero.

(i) The following implications hold in general:

$$
(4) \Longrightarrow(3) \Longrightarrow(2) \Longrightarrow(1) \text {. }
$$

(ii) If $B$ is simple, then the following equivalences hold:

$$
(3) \Longleftrightarrow(2) \Longleftrightarrow(1) \text {. }
$$

(iii) If $B$ is simple and has slow dimension growth, then all the conditions (1), (2), (3), and (4) are equivalent.

Proof. The statements (i) and (ii) are proved in Theorem 1.3 of [2]. The statement (iii) is an immediate consequence of the statement (ii) and Theorem 2 of [12].

An $\mathrm{AH} C^{*}$-algebra $B$ is said to have small eigenvalue variation (in the sense of Bratteli and Elliott, [3]) if $B$ satisfies statement (3) of Theorem 4.

2.4. Rearrange Eigenvalue Pattern. In order to evaluate the eigenvalue variation [3] of a diagonal element $a=$ $\operatorname{diag}\left(a_{1}, \ldots, a_{n}\right)$ in $M_{n}(C(X))$, we need to rearrange the $a_{i}$ so that the obtained one $b=\operatorname{diag}\left(b_{1}, \ldots, b_{n}\right)$ with $b_{1} \leq b_{2} \leq \cdots \leq$ $b_{n}$ has the same eigenvalue variation of $a$.

The eigenvalue variations of two unitary equivalent selfadjoint elements are equal since their eigenvalues are the same. However, the converse need not be true in general. More precisely, there is a self-adjoint element $h$ in $M_{2}\left(C\left(S^{4}\right)\right)$ which is not unitarily equivalent to $\operatorname{diag}\left(\lambda_{1}, \lambda_{2}\right)$ but the eigenvalue variations of both elements are equal, where $\lambda_{i}$ is the $i$ th lowest eigenvalue of $h$ counted with multiplicity [13, Section 2]. In general, given a self-adjoint element $h \in M_{n}(C(X))$, for each $x \in X$, there is a (point-wise) unitary $u(x) \epsilon$ $M_{n}$ such that $h(x)=u(x) \operatorname{diag}\left(\lambda_{1}(x), \lambda_{2}(x), \ldots, \lambda_{n}(x)\right) u^{*}(x)$, where $\lambda_{i}(x)$ is the $i$ th lowest eigenvalue of $h(x)$ counted with multiplicity. Denote by $\operatorname{EV}(h)$ the eigenvalue variation of $h$, then $\operatorname{EV}(h)=\operatorname{EV}\left(\operatorname{diag}\left(\lambda_{1}, \lambda_{2}, \ldots, \lambda_{n}\right)\right)$ but $u(x)$ need not be continuous. The fact is that if $u(x)$ is continuous for any self-adjoint $h$ in $M_{n}(C(X))$, then $\operatorname{dim}(X)$ is less than 3 [13]. However, when replacing the equality "=" by some approximation " $\approx$ " and in some spacial cases (diagonal elements) discussed below, we can get such a continuous unitary without any hypothesis on dimension. Let us see the idea via the following example.

Let $h=\operatorname{diag}(x, 1-x) \in M_{2}(C[0,1])$. Given any $1 / 2>\varepsilon>$ 0 . By $\left[4\right.$, Lemma 2.5], there is a unitary $u \in M_{2}(C[0,1])$ such that

(i) $u(x)=1 \in M_{2}$, for all $x \in[0,1 / 2-\varepsilon]$,

(ii) $u(x)=\left(\begin{array}{ll}0 & 1 \\ 1 & 0\end{array}\right)$, for all $x \in[1 / 2+\varepsilon, 1]$.
Denote by $\lambda_{1}$ and $\lambda_{2}$ the eigenvalue maps of $h$; that is,

$$
\lambda_{1}(x)=\left\{\begin{array}{ll}
x & \text { if } x \leq \frac{1}{2} \\
1-x & \text { if } x>\frac{1}{2},
\end{array} \quad \lambda_{2}(x)= \begin{cases}1-x & \text { if } x \leq \frac{1}{2} \\
x & \text { if } x>\frac{1}{2}\end{cases}\right.
$$

Then $\operatorname{EV}(h)=\operatorname{EV}\left(\operatorname{diag}\left(\lambda_{1}, \lambda_{2}\right)\right)=1 / 2$.

It is straightforward to check that $\left\|u h u^{*}-\operatorname{diag}\left(\lambda_{1}, \lambda_{2}\right)\right\| \leq$ $\varepsilon$

Lemma 5. Let $X$ be a connected compact Hausdorff space and $h=\operatorname{diag}\left(f_{1}, f_{2}, \ldots, f_{n}\right)$ a self-adjoint element in $M_{n}(C(X))$, where $f_{1}, f_{2}, \ldots, f_{n}$ are continuous maps from $X$ to $\mathbb{R}$. For any positive number $\varepsilon$, there is a unitary $u \in M_{n}(C(X))$ such that

$$
\left\|u h u^{*}-\operatorname{diag}\left(\lambda_{1}, \lambda_{2}, \ldots, \lambda_{n}\right)\right\|<\varepsilon,
$$

where the $\lambda_{i}(x)$ is the ith lowest eigenvalue of $h(x)$ counted with multiplicity for every $x \in X$.

Proof. If $f_{1} \leq f_{2} \leq \cdots \leq f_{n}$, then the unitary $u$ is just the identity of $M_{n}$ and $\lambda_{i}=f_{i}$. Therefore, to prove the lemma, we, roughly speaking, only need to rearrange the given family $\left\{f_{1}, f_{2}, \ldots, f_{n}\right\}$ to obtain an increasing ordered family. For $n=$ 1 , the lemma is obvious. Otherwise, using the idea of bubble sort, we can reduce to the case $n=2$.

Let $Z=\left(\lambda_{1}-\lambda_{2}\right)^{-1}(-\varepsilon / 2, \varepsilon / 2)$. Set $E=\left\{x \in X: f_{1}(x) \leq\right.$ $\left.f_{2}(x)\right\} \cap(X \backslash Z)$ and $F=\left\{x \in X: f_{1}(x) \geq f_{2}(x)\right\} \cap(X \backslash Z)$.

It is clear that $E$ and $F$ are disjoint closed sets and $X=$ $E \cup F \cup Z$. We have $\lambda_{1}(x)=\min \left\{f_{1}(x), f_{2}(x)\right\}$ and $\lambda_{2}(x)=$ $\max \left\{f_{1}(x), f_{2}(x)\right\}$ for all $x \in X$. If $E(F)$ is empty, then the unitary $u$ can be chosen as $\left(\begin{array}{ll}0 & 1 \\ 1 & 0\end{array}\right)\left(1 \in M_{2}\right.$, resp.). Thus, we can assume both $E$ and $F$ are nonempty. By Urysohn's Lemma, there is a continuous map $\mu: X \rightarrow[0,1]$ such that $\mu$ is equal to 0 on $E$ and 1 on $F$. Since the space of unitary matrices of $M_{2}$ is path connected, there is a unitary path $p$ linking

$$
p(0)=1 \text { to } p(1)=\left(\begin{array}{ll}
0 & 1 \\
1 & 0
\end{array}\right) \text {. }
$$

Consequently, $u=p \circ \mu$ is a unitary in $M_{2}(C(X))$ and $u(x) h(x) u^{*}(x)=\operatorname{diag}\left(\lambda_{1}(x), \lambda_{2}(x)\right)$ for all $x \in E \cup F$.

For $x \in X \backslash(E \cup F)=Z$, we have

$$
\left|\lambda_{1}(x)-\lambda_{2}(x)\right|<\frac{\varepsilon}{2}, \quad\left|f_{i}(x)-\lambda_{1}(x)\right|<\frac{\varepsilon}{2}, \quad i=1,2 \text {. }
$$

Hence,

$$
\begin{gathered}
\left\|\operatorname{diag}\left(\lambda_{1}(x), \lambda_{2}(x)\right)-\operatorname{diag}\left(\lambda_{1}(x), \lambda_{1}(x)\right)\right\|<\frac{\varepsilon}{2}, \\
\left\|h(x)-\operatorname{diag}\left(\lambda_{1}(x), \lambda_{1}(x)\right)\right\|<\frac{\varepsilon}{2} .
\end{gathered}
$$

On account to (23) we have

$$
\begin{aligned}
& \left\|u(x) h(x) u^{*}(x)-\operatorname{diag}\left(\lambda_{1}(x), \lambda_{2}(x)\right)\right\| \\
& \leq\left\|u(x)\left[h(x)-\operatorname{diag}\left(\lambda_{1}(x), \lambda_{1}(x)\right)\right] u^{*}(x)\right\| \\
& \quad+\left\|\operatorname{diag}\left(\lambda_{1}(x), \lambda_{1}(x)\right)-\operatorname{diag}\left(\lambda_{1}(x), \lambda_{2}(x)\right)\right\| \\
& \quad<\varepsilon .
\end{aligned}
$$


Therefore,

$$
\left\|u h u^{*}-\operatorname{diag}\left(\lambda_{1}, \lambda_{2}\right)\right\|<\varepsilon .
$$

The main result of this section as follows.

Theorem 6. Given an $A H$ algebra $A=\underline{\lim }\left(A_{i}, \phi_{i}\right)$, where the $\phi_{i}$ are diagonal $*$-homomorphisms from $\vec{A}_{i}$ to $A_{i+1}$, where $A_{i}=\oplus_{t=1}^{k_{i}} M_{n_{i t}}\left(C\left(X_{i t}\right)\right)$ and the $X_{i t}$ are connected compact Hausdorff spaces. If $A$ has small eigenvalue variation in the sense of Bratteli and Elliott, then A has the LP property.

Proof. By Corollary 2, it suffices to show that $\phi_{i \infty}(f) \in L(A)$ for every real-valued function $f \in C\left(X_{i t}\right)$. By the same argument in the proof of Theorem 1 , we can assume that each $A_{t}$ has only one component; that is, $A_{t}=M_{n_{t}}\left(C\left(X_{t}\right)\right)$. Let $\varepsilon>0$ be arbitrary. Since $A$ has small eigenvalue variation in the sense of Bratteli and Elliott, there is an integer $j \geq i$ such that $\operatorname{EV}\left(\phi_{i j}(f)\right)<\varepsilon$. Let $\left\{\mu_{1}, \ldots, \mu_{n}\right\}$ be the eigenvalue pattern of $\phi_{i j}\left(n=n_{j} / n_{i}\right)$. Then,

$$
\begin{aligned}
\phi_{i j}(f) & =\phi_{i j}(\operatorname{diag}(f, 0)) \\
& =\operatorname{diag}\left(f \circ \mu_{1}, 0, f \circ \mu_{2}, 0, \ldots, f \circ \mu_{n}, 0\right) \\
& =v \operatorname{diag}\left(f_{1}, f_{2}, \ldots, f_{n}, 0\right) v^{*},
\end{aligned}
$$

where $f_{i}=f \circ \mu_{i}$ and $v$ is the permutation matrix in $M_{n_{j}}$ moving all the zero to the bottom left-hand corner. Note that

$$
\operatorname{EV}\left(\phi_{i j}(f)\right)=\operatorname{EV}\left(\operatorname{diag}\left(f_{1}, f_{2}, \ldots, f_{n}, f_{n+1}\right)\right)
$$

where $f_{n+1}(x)=0$ for all $x \in X_{j}$. By Lemma 5, there exists a unitary $u \in M_{n+1}\left(C\left(X_{j}\right)\right)$ and eigenvalue maps $\lambda_{1} \leq \lambda_{2} \leq \cdots \leq \lambda_{n+1}$ of $\operatorname{diag}\left(f_{1}, f_{2}, \ldots, f_{n}, f_{n+1}\right)$ such that

$$
\left\|u \operatorname{diag}\left(f_{1}, f_{2}, \ldots, f_{n}, f_{n+1}\right) u^{*}-\operatorname{diag}\left(\lambda_{1}, \ldots, \lambda_{n+1}\right)\right\|<\varepsilon .
$$

$$
\begin{aligned}
& \text { Put } \\
& \delta_{i}=\frac{1}{2}\left(\max _{x \in X_{j}} \lambda_{i}(x)+\min _{x \in X_{j}} \lambda_{i}(x)\right), \quad i=1,2, \ldots, n+1 .
\end{aligned}
$$

Then for any $i$, we have

$$
\max _{x \in X_{j}} \lambda_{i}(x)-\min _{x \in X_{j}} \lambda_{i}(x) \leq \operatorname{EV}\left(\phi_{i j}(f)\right)<\varepsilon
$$

and so

$$
\left|\lambda_{i}(x)-\delta_{i}\right|<\varepsilon, \quad \forall x \in X_{j}
$$

Thus,

$$
\left\|u \operatorname{diag}\left(f_{1}, f_{2}, \ldots, f_{n}, f_{n+1}\right) u^{*}-\sum_{i=1}^{n+1} \delta_{i} e_{i i}\right\|<2 \varepsilon,
$$

where $\left\{e_{i j}\right\}$ is the standard basis of $M_{n+1}$. This implies that

$$
\left\|\phi_{i j}(f)-b\right\|<2 \varepsilon
$$

where $b=v^{*} \operatorname{diag}\left(u^{*}\left(\sum_{i=1}^{n+1} \delta_{i} e_{i i}\right) u, 0\right) v$ is a linear combination of projections in $A_{j}$.

Therefore,

$$
\phi_{i \infty}(f) \in L(A) .
$$

\subsection{Another Form of Theorem 6}

Lemma 7. Let $B$ be a $C^{*}$-algebra, and $p$ and $q$ projections in $B$. If $p$ and $q$ are Murray-von Neumann equivalent, then $p B p$ is isomorphic to $q \mathrm{~Bq}$.

In particular, if $B=M_{n}(C(X))$ (where $X$ is a connected compact Hausdorff space) and $q$ is a constant projection of rank $m$ in $B$, then $q B q$ is $*$-isomorphic to $M_{m}(C(X))$.

Proof. By assumption, there exists a partial isometry $v$ such that $p=v^{*} v$ and $q=v v^{*}$. Let us consider the following maps:

$$
\begin{array}{ll}
\phi(x)=v x v^{*} & (x \in p B p), \\
\psi(y)=v^{*} y v & (y \in q B q) .
\end{array}
$$

It is straightforward to check that the compositions of $\phi$ and $\psi$ are the identity maps.

In the case $B=M_{n}(C(X))$ and $q$ is a constant projection of rank $m$ in $B$, we have $q B q=M_{m}(C(X))$. Therefore, $p B p$ is *-isomorphic to $M_{m}(C(X))$.

Theorem 8 (another form of Theorem 6). Let $A=\lim \left(A_{i}, \phi_{i}\right)$ be a diagonal AH algebra, where the $p_{i t}$ are projections in $M_{n_{i t}}\left(C\left(X_{i t}\right)\right), A_{i}=\oplus_{t=1}^{k_{i}} p_{i t} M_{n_{i t}}\left(C\left(X_{i t}\right)\right) p_{i t}$, and the $\phi_{i}$ are unital diagonal. Suppose that each projection $p_{1 t}$ is Murray-von Neumann equivalent to some constant projection in $A_{1}$. Then $A$ has the LP property provided that $A$ has small eigenvalue variation in the sense of Bratteli and Elliott.

Proof. We can assume that $A_{i}=p_{i} M_{n_{i}}\left(C\left(X_{i}\right)\right) p_{i}$, for all $i$. It is easy to see that $p_{1}$ is Murray-von Neumann equivalent to $q_{1}=e_{11}+e_{22}+\cdots+e_{m_{1}}$, where $m_{1}$ is the rank of $p_{1}$. For $i>1$, define $q_{i}=\phi_{i-1}\left(q_{i-1}\right)$. Then $q_{i}=\phi_{1 i}\left(q_{1}\right)$ is constant, since $q_{1}$ is constant and $\phi_{1 i}$ is diagonal. Let us denote by $m_{i}$ the rank of $q_{i}$, then $m_{i+1} \mid m_{i}$. By Lemma 7, there are $*$-isomorphisms $\Theta_{i}$ from $p_{i} M_{n_{i}}\left(C\left(X_{i}\right)\right) p_{i}$ to $q_{i} M_{n_{i}}\left(C\left(X_{i}\right)\right) q_{i}=M_{m_{i}}(C(X))$ such that

$$
\Theta_{i}(a)=v_{i} a v_{i}^{*},
$$

where $p_{1}=v_{1}^{*} v_{1} \sim v_{1} v_{1}^{*}=q_{1}$ and $v_{i}=\phi_{1 i}\left(v_{1}\right)$. Since $\phi_{i}$ is diagonal, there exists its extension $\widetilde{\phi}_{i}$ which is a diagonal *-homomorphism from $M_{n_{i}}\left(C\left(X_{i}\right)\right)$ to $M_{n_{i+1}}\left(C\left(X_{i+1}\right)\right)$. 
Let $\psi_{i}$ be the restriction of $\tilde{\phi}_{i}$ on $q_{i} M_{n_{i}}\left(C\left(X_{i}\right)\right) q_{i}$. Then $\psi_{i}\left(q_{i}\right)=q_{i+1}$. Therefore, the map $\psi_{i}$ can be viewed as the map from $q_{i} M_{n_{i}}\left(C\left(X_{i}\right)\right) q_{i}$ to $q_{i+1} M_{n_{i+1}}\left(C\left(X_{i+1}\right)\right) q_{i+1}$ and so $\underset{\lim }{\longrightarrow}\left(M_{m_{i}}\left(C\left(X_{i}\right)\right), \psi_{i}\right)$ is a diagonal AH-algebra.

On the other hand, it is straightforward to check that $\Theta_{i+1} \circ \phi_{i}=\psi_{i} \circ \Theta_{i}$ and hence $A=\underline{\lim }\left(M_{m_{i}}\left(C\left(X_{i}\right)\right), \psi_{i}\right)$. By Theorem 6, $A$ has the LP property.

2.6. Examples. In some special cases, small eigenvalue variation in the sense of Bratteli and Elliott and the LP property are equivalent.

Example 9. Let $A=\lim \left(M_{v(n)}(C(X)), \phi_{n}\right)$ be a Goodearl algebra [6] and $\omega_{t, 1}$ the weighted identity ratio for $\phi_{t, 1}$. Suppose that $X$ is not totally disconnected and has finitely many connected components, then the following statements are equivalent.

(i) A has real rank zero.

(ii) $\lim _{t \rightarrow \infty} \omega_{t, 1}=0$.

(iii) A has small eigenvalue variation in the sense of Bratteli and Elliott.

(iv) $A$ has the LP property.

Proof. Indeed, (i) and (ii) are equivalent by [6, Theorem 9]. The implication (i) $\Rightarrow$ (iii) follows from [2, Theorem 1.3]. By [14, Theorem 2.6], (i) implies (iv). Using Theorem 6 we get the implication (iii) $\Rightarrow$ (iv). Finally, (iv) implies (ii) by [6, Theorem 6].

In general, the LP property cannot imply small eigenvalue variation in the sense of Bratteli and Elliott nor real rank zero. For example, let $A$ be a simple AH algebra with slow dimension growth and $H$ be a simple hereditary $C^{*}$ subalgebra of $A$. By [5, Theorem 3.5], $H$ has nontrivial projections. Hence, $H \otimes K$ has the LP property by [1, Corollary $5]$. However, $A$ has real rank zero if and only if it has small eigenvalue variation in the sense of Bratteli and Elliott [3]. This means that we can choose $H$ with real rank nonzero such that $H \otimes K$ has the LP property and does not have small eigenvalue variation in the sense of Bratteli and Elliott.

Looking for examples in the class of diagonal AH algebras, we need the following lemma.

Lemma 10. Let $A$ be a diagonal $A H$ algebra and $K$ be the $C^{*}$ algebra of compact operators on an infinite dimensional Hilbert space. Then the tensor product $A \otimes K$ is again diagonal.

Proof. Let $A=\lim \left(A_{n}, \phi_{n}\right)$ and $K=\lim \left(M_{n}, i_{n}\right)$, where $A_{n}$ is a homogeneous algebra, $\phi_{n}$ is an injective diagonal homomorphism from $A_{n}$ to $A_{n+1}$, and $i_{n}$ is the embedding from $M_{n}$ to $M_{n+1}$ which associates each $a \in M_{n}$ to $\operatorname{diag}(a, 0) \in M_{n+1}$ for each positive integer $n$. Let us consider the inductive limit $\lim _{\longrightarrow}\left(A_{n} \otimes M_{n}, \phi_{n} \otimes i_{n}\right)$. For each integer $n \geq 1$, denote by $i_{n, \infty}$ and $\phi_{n, \infty}$ the homomorphisms from $M_{n}$ and $A_{n}$ to $K$ and $A$ in the inductive limit of $K$ and $A$, respectively. Then

$$
\left(\phi_{n+1, \infty} \otimes i_{n+1, \infty}\right) \circ\left(\phi_{n} \otimes i_{n}\right)=\phi_{n, \infty} \otimes i_{n, \infty} .
$$

Hence, by the universal property of inductive limit, there exists a unique homomorphism $\Phi$ from $\lim _{\longrightarrow}\left(A_{n} \otimes M_{n}, \phi_{n} \otimes i_{n}\right)$ to $A \otimes K$ such that

$$
\Phi \circ\left(\phi_{n} \otimes i_{n}\right)=\left(\phi_{n, \infty} \otimes i_{n, \infty}\right) .
$$

It is straightforward to check that the image of $\Phi$ is dense in $A \otimes K$ and since all the maps $\phi_{n}$ and $i_{n}$ are injective, we have $\lim _{\longrightarrow}\left(A_{n} \otimes M_{n}, \phi_{n} \otimes i_{n}\right)$ is $A \otimes K$. Furthermore, for each $n$, we identify an element $a \otimes b$ in $A_{n} \otimes M_{n}$ with the matrix $\left(a_{i j} b\right)$ in $M_{n}\left(A_{n}\right)$, where $a=\left(a_{i j}\right) \in M_{n}$ and $b \in A_{n}$. By interchanging rows and columns (independent of $a \otimes b$ ) of $\left(\phi_{n} \otimes i_{n}\right)(a \otimes b)$, we obtain $\operatorname{diag}\left(a \otimes b \circ \lambda_{1}, \ldots, a \otimes b \circ \lambda_{m}, 0\right)$, where $\lambda_{1}, \ldots, \lambda_{m}$ are the eigenvalue maps of $\phi_{n}$. This means that there is a permutation matrix $u_{n} \in M_{n+1}\left(A_{n+1}\right)$ such that $u_{n} \phi_{n} \otimes i_{n} u_{n}^{*}$ is diagonal. The fact is that the inductive limit is unchanged under unitary equivalence; that is,

$$
\lim _{\longrightarrow}\left(A_{n} \otimes M_{n}, \phi_{n} \otimes i_{n}\right)=\lim _{\longrightarrow}\left(A_{n} \otimes M_{n}, u_{n} \phi_{n} \otimes i_{n} u_{n}^{*}\right)
$$

Hence, $\lim _{\longrightarrow}\left(A_{n} \otimes M_{n}, \phi_{n} \otimes i_{n}\right)$ is diagonal.

Example 11. Let $B$ be a simple unital diagonal $\mathrm{AH}$ algebra with real rank one without the LP property (e.g., take a Goodearl algebra, see Example 9), then $B \otimes K$ is a diagonal $\mathrm{AH}$ algebra of real rank one with the LP property.

Proof. By Lemma $10, B \otimes K$ is a diagonal $\mathrm{AH}$ algebra. The real rank of $B \otimes K$ is one since that of $B$ is nonzero. Since $B$ is unital, $B \otimes K$ has a nontrivial projection. By [1, Corollary 5], $B \otimes K$ has the LP property.

\section{The LP Property for an Inclusion of Unital $C^{*}$-Algebras}

3.1. Examples. In this subsection, we will show that the LP property is not stable under the fixed point operation via the given examples. Firstly, we could observe the following example which shows that the LP property is not stable under the hereditary subalgebra.

Lemma 12. Let $A$ be a projectionless simple unital $C^{*}$-algebra with a unique tracial state. Then for any $n \in \mathbb{N}$ with $n>1$, $M_{n}(A)$ has the LP property.

Proof. Note that $M_{n}(A)$ has also a unique tracial state.

Since $A$ is unital, $M_{n}(A)$ has a nontrivial projection. Then by $[1$, Corollary 5$], M_{n}(A)$ has the LP property.

Remark 13. Let $A$ be the Jiang-Su algebra. Then we know that $R R(A)=1$ [14]. Since $M_{n}(A)$ is an AH algebra without real rank zero, $R R\left(M_{n}(A)\right)=1$. But from Lemma $12, M_{n}(A)$ has the LP property. 
Using this observation, we can construct a $C^{*}$-algebra with the LP property such that the fixed point algebra does not have the LP property.

Example 14. A simple unital AI algebra $A$ in [15, Example 9], which comes from Thomsen's construction, has two extremal tracial states; so by [16, Theorem 4.4], A does not have the LP property. There is a symmetry $\alpha$ on $A$ constructed by Elliott such that $A \rtimes_{\alpha} \mathbb{Z} / 2 \mathbb{Z}$ is a UHF algebra. Since the fixed point algebra $\left(A \rtimes_{\alpha} \mathbb{Z} / 2 \mathbb{Z}\right)^{\beta}=A$, where $\beta$ is the dual action of $\alpha$. This shows that there is a simple unital $C^{*}$-algebra $B$ with the LP property such that the fixed point algebra $B^{\beta}$ does not have the LP property.

3.2. $C^{*}$-Index Theory. According to Example 14, there is a faithful conditional expectation $E: B \rightarrow B^{\beta}$. We extend this observation to an inclusion of unital $C^{*}$-algebras with a finite Watatani index as follows.

In this section we recall the $C^{*}$-basic construction defined by Watatani.

Definition 15. Let $A \supset P$ be an inclusion of unital $C^{*}$-algebras with a conditional expectation $E$ from $A$ to $P$.

(1) A quasi-basis for $E$ is a finite set $\left\{\left(u_{i}, v_{i}\right)\right\}_{i=1}^{n} \subset A \times A$ such that for every $a \in A$,

$$
a=\sum_{i=1}^{n} u_{i} E\left(v_{i} a\right)=\sum_{i=1}^{n} E\left(a u_{i}\right) v_{i}
$$

(2) When $\left\{\left(u_{i}, v_{i}\right)\right\}_{i=1}^{n}$ is a quasi-basis for $E$, we define index $E$ by

$$
\text { Index } E=\sum_{i=1}^{n} u_{i} v_{i}
$$

When there is no quasi-basis, we write Index $E=\infty$. index $E$ is called the Watatani index of $E$.

Remark 16. We give several remarks about the above definitions.

(1) Index $E$ does not depend on the choice of the quasibasis in the above formula, and it is a central element of $A$ [17, Proposition 1.2.8].

(2) Once we know that there exists a quasi-basis, we can choose one of the form $\left\{\left(w_{i}, w_{i}^{*}\right)\right\}_{i=1}^{m}$, which shows that Index $E$ is a positive element [17, Lemma 2.1.6].

(3) By the above statements, if $A$ is a simple $C^{*}$-algebra, then Index $E$ is a positive scalar.

(4) If Index $E<\infty$, then $E$ is faithful; that is, $E\left(x^{*} x\right)=0$ implies $x=0$ for $x \in A$.

Next we recall the $C^{*}$-basic construction defined by Watatani.

Let $E: A \rightarrow P$ be a faithful conditional expectation. Then $A_{P}(=A)$ is a pre-Hilbert module over $P$ with a $P$ valued inner product

$$
\langle x, y\rangle_{P}=E\left(x^{*} y\right), \quad x, y \in A_{P} .
$$

We denote by $\mathscr{E}_{E}$ and $\eta_{E}$ the Hilbert $P$ module completion of $A$ by the norm $\|x\|_{P}=\left\|\langle x, x\rangle_{P}\right\|^{1 / 2}$ for $x$ in $A$ and the natural inclusion map from $A$ to $\mathscr{E}_{E}$. Then $\mathscr{E}_{E}$ is a Hilbert $C^{*}$-module over $P$. Since $E$ is faithful, the inclusion map $\eta_{E}$ from $A$ to $\mathscr{E}_{E}$ is injective. Let $L_{P}\left(\mathscr{E}_{E}\right)$ be the set of all (right) $P$ module homomorphisms $T: \mathscr{E}_{E} \rightarrow \mathscr{E}_{E}$ with an adjoint right $P$ module homomorphism $T^{*}: \mathscr{E}_{E} \rightarrow \mathscr{E}_{E}$ such that

$$
\langle T \xi, \zeta\rangle=\left\langle\xi, T^{*} \zeta\right\rangle, \quad \xi, \zeta \in \mathscr{E}_{E}
$$

Then $L_{P}\left(\mathscr{E}_{E}\right)$ is a $C^{*}$-algebra with the operator norm $\|T\|=$ $\sup \{\|T \xi\|:\|\xi\|=1\}$. There is an injective $*$-homomorphism $\lambda: A \rightarrow L_{P}\left(\mathscr{E}_{E}\right)$ defined by

$$
\lambda(a) \eta_{E}(x)=\eta_{E}(a x)
$$

for $x \in A_{P}$ and $a \in A$, so that $A$ can be viewed as a $C^{*}$ subalgebra of $L_{P}\left(\mathscr{E}_{E}\right)$. Note that the map $e_{P}: A_{P} \rightarrow A_{P}$ defined by

$$
e_{P} \eta_{E}(x)=\eta_{E}(E(x)), \quad x \in A_{P}
$$

is bounded and thus it can be extended to a bounded linear operator, denoted by $e_{P}$ again, on $\mathscr{E}_{E}$. Then $e_{P} \in L_{P}\left(\mathscr{E}_{E}\right)$ and $e_{P}=e_{P}^{2}=e_{P}^{*}$; that is, $e_{P}$ is a projection in $L_{P}\left(\mathscr{E}_{E}\right)$. A projection $e_{P}$ is called the Jones projection of $E$.

The (reduced) $C^{*}$-basic construction is a $C^{*}$-subalgebra of $L_{P}\left(\mathscr{E}_{E}\right)$, defined as

$$
C_{r}^{*}\left\langle A, e_{P}\right\rangle=\overline{\operatorname{span}\left\{\lambda(x) e_{P} \lambda(y) \in L_{P}\left(\mathscr{E}_{E}\right): x, y \in A\right\}^{\|\cdot\|} .}
$$

Remark 17. Watatani proved the following in [17].

(1) Index $E$ is finite if and only if $C_{r}^{*}\left\langle A, e_{P}\right\rangle$ has the identity (equivalently $C_{r}^{*}\left\langle A, e_{P}\right\rangle=L_{P}\left(\mathscr{E}_{E}\right)$ ) and there exists a constant $c>0$ such that $E\left(x^{*} x\right) \geq c x^{*} x$ for $x \in A$; that is, $\|x\|_{P}^{2} \geq c\|x\|^{2}$ for $x$ in $A$ by [17, Proposition 2.1.5]. Since $\|x\| \geq\|x\|_{P}$ for $x$ in $A$, if index $E$ is finite, then $\mathscr{E}_{E}=A$.

(2) If index $E$ is finite, then each element $z$ in $C_{r}^{*}\left\langle A, e_{P}\right\rangle$ has a form

$$
z=\sum_{i=1}^{n} \lambda\left(x_{i}\right) e_{P} \lambda\left(y_{i}\right)
$$

for some $x_{i}$ and $y_{i}$ in $A$.

(3) Let $C_{\max }^{*}\left\langle A, e_{P}\right\rangle$ be the unreduced $C^{*}$-basic construction defined in Definition 2.2.5 of [17], which has the certain universality (cf.(5) below). If index $E$ is finite, then there exists an isomorphism from $C_{r}^{*}\left\langle A, e_{p}\right\rangle$ to $C_{\max }^{*}\left\langle A, e_{P}\right\rangle[17$, Proposition 2.2.9]. Therefore, we can identify $C_{r}^{*}\left\langle A, e_{P}\right\rangle$ with $C_{\max }^{*}\left\langle A, e_{P}\right\rangle$. So we call $C_{r}^{*}\left\langle A, e_{P}\right\rangle$ the $C^{*}$-basic construction and denote it by $C^{*}\left\langle A, e_{P}\right\rangle$. Moreover, we identify $\lambda(A)$ with $A$ in $C^{*}\left\langle A, e_{p}\right\rangle\left(=C_{r}^{*}\left\langle A, e_{P}\right\rangle\right)$, and we define it as

$$
C^{*}\left\langle A, e_{p}\right\rangle=\left\{\sum_{i=1}^{n} x_{i} e_{P} y_{i}: x_{i}, y_{i} \in A, n \in \mathbb{N}\right\} \text {. }
$$


(4) The $C^{*}$-basic construction $C^{*}\left\langle A, e_{p}\right\rangle$ is isomorphic to $q M_{n}(P) q$ for some $n \in \mathbb{N}$ and projection $q \in M_{n}(P)$ [17, Lemma 3.3.4]. If index $E$ is finite, then index $E$ is a central invertible element of $A$ and there is the dual conditional expectation $\hat{E}$ from $C^{*}\left\langle A, e_{P}\right\rangle$ to $A$ such that

$$
\hat{E}\left(x e_{P} y\right)=(\operatorname{Index} E)^{-1} x y \quad \text { for } x, y \in A
$$

by [17, Proposition 2.3.2]. Moreover, $\hat{E}$ has a finite index and faithfulness. If $A$ is simple unital $C^{*}$ algebra, index $E \in A$ by Remark 16(4). Hence index $E=$ index $\hat{E}$ by [17, Proposition 2.3.4].

(5) Suppose that index $E$ is finite and $A$ acts on a Hilbert space $\mathscr{H}$ faithfully and $e$ is a projection on $\mathscr{H}$ such that $e a e=E(a) e$ for $a \in A$. If a map $P \ni x \mapsto x e \in$ $B(\mathscr{H})$ is injective, then there exists an isomorphism $\pi$ from the norm closure of a linear span of $A e A$ to $C^{*}\left\langle A, e_{P}\right\rangle$ such that $\pi(e)=e_{P}$ and $\pi(a)=a$ for $a \in A$ [17, Proposition 2.2.11].

3.3. Rokhlin Property for an Inclusion of Unital $C^{*}$-Algebras. For a $C^{*}$-algebra $A$, we set

$$
\begin{gathered}
c_{0}(A)=\left\{\left(a_{n}\right) \in l^{\infty}(\mathbb{N}, A): \lim _{n \rightarrow \infty}\left\|a_{n}\right\|=0\right\}, \\
A^{\infty}=\frac{l^{\infty}(\mathbb{N}, A)}{c_{0}(A)} .
\end{gathered}
$$

We identify $A$ with the $C^{*}$-subalgebra of $A^{\infty}$ consisting of the equivalence classes of constant sequences and set

$$
A_{\infty}=A^{\infty} \cap A^{\prime}
$$

For an automorphism $\alpha \in \operatorname{Aut}(A)$, we denote by $\alpha^{\infty}$ and $\alpha_{\infty}$ the automorphisms of $A^{\infty}$ and $A_{\infty}$ induced by $\alpha$, respectively.

Izumi defined the Rokhlin property for a finite group action in [18, Definition 3.1] as follows.

Definition 18. Let $\alpha$ be an action of a finite group $G$ on a unital $C^{*}$-algebra $A$. $\alpha$ is said to have the Rokhlin property if there exists a partition of unity $\left\{e_{g}\right\}_{g \in G} \subset A_{\infty}$ consisting of projections satisfying

$$
\left(\alpha_{g}\right)_{\infty}\left(e_{h}\right)=e_{g h} \quad \text { for } g, h \in G
$$

We call $\left\{e_{g}\right\}_{g \in G}$ the Rokhlin projections.

Let $A \supset P$ be an inclusion of unital $C^{*}$-algebras. For a conditional expectation $E$ from $A$ to $P$, we denote by $E^{\infty}$ the natural conditional expectation from $A^{\infty}$ to $P^{\infty}$ induced by $E$. If $E$ has a finite index with a quasi-basis $\left\{\left(u_{i}, v_{i}\right)\right\}_{i=1}^{n}$, then $E^{\infty}$ also has a finite index with a quasi-basis $\left\{\left(u_{i}, v_{i}\right)\right\}_{i=1}^{n}$ and $\operatorname{Index}\left(E^{\infty}\right)=\operatorname{Index} E$.

Motivated by Definition 18, Kodaka et al. introduced the Rokhlin property for an inclusion of unital $C^{*}$-algebras with a finite index [19].
Definition 19. A conditional expectation $E$ of a unital $C^{*}-$ algebra $A$ with a finite index is said to have the Rokhlin property if there exists a projection $e \in A_{\infty}$ satisfying

$$
E^{\infty}(e)=(\operatorname{Index} E)^{-1} \cdot 1
$$

and a map $A \ni x \mapsto x e$ is injective. We call $e$ a Rokhlin projection.

The following result states that the Rokhlin property of an action in the sense of Izumi implies that the canonical conditional expectation from a given simple $C^{*}$-algebra to its fixed point algebra has the Rokhlin property in the sense of Definition 19.

Proposition 20 (see [19]). Let $\alpha$ be an action of a finite group $G$ on a unital $C^{*}$-algebra $A$ and $E$ the canonical conditional expectation from $A$ to the fixed point algebra $P=A^{\alpha}$ defined by

$$
E(x)=\frac{1}{\# G} \sum_{g \in G} \alpha_{g}(x), \quad \text { for } x \in A \text {, }
$$

where $\# G$ is the order of $G$. Then $\alpha$ has the Rokhlin property if and only if there is a projection $e \in A_{\infty}$ such that $E^{\infty}(e)=$ $(1 / \# G) \cdot 1$, where $E^{\infty}$ is the conditional expectation from $A^{\infty}$ to $P^{\infty}$ induced by $E$.

The following is the key one in the next section.

Proposition 21 (see [19] and [20, Lemma 2.5]). Let $P \subset A$ be an inclusion of unital $C^{*}$-algebras and $E$ a conditional expectation from A to $P$ with a finite index. If $E$ has the Rokhlin property with a Rokhlin projection $e \in A_{\infty}$, then there is a unital linear map $\beta: A^{\infty} \rightarrow P^{\infty}$ such that for any $x \in A^{\infty}$ there exists the unique element $y$ of $P^{\infty}$ such that $x e=y e=$ $\beta(x) e$ and $\beta\left(A^{\prime} \cap A^{\infty}\right) \subset P^{\prime} \cap P^{\infty}$. In particular, $\beta_{\left.\right|_{A}}$ is a unital injective $*$-homomorphism and $\beta(x)=x$ for all $x \in P$.

The following is contained in [19, Proposition 3.4]. But we give it for self-contained.

Proposition 22. Let $P \subset A$ be an inclusion of unital $C^{*}$ algebras and $E$ conditional expectation from $A$ to $P$ with a finite index. Suppose that $A$ is simple. Consider the basic construction

$$
P \subset A \subset C^{*}\left\langle A, e_{P}\right\rangle(:=B) \subset C^{*}\left\langle B, e_{A}\right\rangle\left(:=B_{1}\right) .
$$

If $E: A \rightarrow P$ has the Rokhlin property with a Rokhlin projection $e \in A_{\infty}$, then the double dual conditional expectation $\hat{\hat{E}}\left(:=E_{B}\right): C^{*}\left\langle B, e_{A}\right\rangle \rightarrow B$ has the Rokhlin property.

Proof. Note that from Remark 17(4) and [19, Corollary 3.8], $C^{*}$-algebras $C^{*}\left\langle A, e_{P}\right\rangle$ and $C^{*}\left\langle B, e_{A}\right\rangle$ are simple. 
Since $e_{P} e e_{P}=E^{\infty}(e) e_{P}=(\operatorname{Index} E)^{-1} e_{p},(\operatorname{Index} E) e e_{p} e \leq$ $e$, and

$$
\begin{aligned}
\hat{E}^{\infty}\left(e-(\operatorname{Index} E) e e_{p} e\right) & =e-(\operatorname{Index} E) e \hat{E}^{\infty}\left(e_{P}\right) e \\
& =e-e=0,
\end{aligned}
$$

we have $e=(\operatorname{Index} E) e e_{P} e$. Then, for any $x, y \in A$

$$
\begin{aligned}
e\left(x e_{P} y\right) e & =\text { exee }_{P} \text { eye } \\
& =(\operatorname{Index} E)^{-1} \text { exye } \\
& =\hat{E}\left(x e_{P} y\right) e .
\end{aligned}
$$

Hence, from Remark 17(3), we have eze $=\hat{E}(z) e$ for any $z \epsilon$ $C^{*}\left\langle A, e_{p}\right\rangle$.

Let $\left\{\left(w_{i}, w_{i}^{*}\right)\right\} \subset B \times B$ be a quasi-basis for $\hat{E}\left(=E_{A}\right)$ and $e_{A}$ be the Jones projection of $\hat{E}$. Set $g=\sum_{i} w_{i} e e_{A} w_{i}^{*} \in B_{1}^{\infty}$. Then $g$ is a projection and $g \in B_{1}^{\prime}$. Indeed, since

$$
\begin{aligned}
g^{2} & =\sum_{i, j} w_{i} e e_{A} w_{i}^{*} w_{j} e e_{A} w_{j}^{*} \\
& =\sum_{i, j} w_{i} e e_{A} \hat{E}\left(w_{i}^{*} w_{j}\right) w_{j}^{*} \\
& =\sum_{i} w_{i} e e_{A}\left(\sum_{j} \hat{E}\left(w_{i}^{*} w_{j}\right) w_{j}^{*}\right) \\
& =\sum_{i} w_{i} e e_{A} w_{i}^{*}=g,
\end{aligned}
$$

$g$ is a projection.

Consider the following:

$$
\begin{aligned}
g e_{A} & =\sum_{i} w_{i} e e_{A} w_{i}^{*} e_{A}=\sum_{i} w_{i} \hat{E}\left(w_{i}^{*}\right) e e_{A} \\
& =e e_{A}, \\
e_{A} g & =e_{A} \sum_{i} w_{i} e e_{A} w_{i}^{*}=\sum_{i} \hat{E}\left(w_{i}\right) e_{A} e w_{i}^{*} \\
& =e_{A} e \sum_{i} \hat{E}\left(w_{i}\right) w_{i}^{*} \\
& =e e_{A}=g e_{A} .
\end{aligned}
$$

Moreover, for any $z \in C^{*}\left\langle A, e_{P}\right\rangle$, we have

$$
\begin{aligned}
g z & =\sum_{i} w_{i} e e_{A} w_{i}^{*} z \\
& =\sum_{i} w_{i} e e_{A}\left(\sum_{j} \hat{E}\left(w_{i}^{*} z w_{j}\right) w_{j}^{*}\right) \\
& =\sum_{i} w_{i} \sum_{j} \hat{E}\left(w_{i}^{*} x z w_{j}\right) e e_{A} w_{j}^{*} \\
& =\sum_{j}\left(\sum_{i} w_{i} \hat{E}\left(w_{i}^{*} z w_{j}\right)\right) e e_{A} w_{j}^{*} \\
& =\sum_{j} z w_{j} e e_{A} w_{j}^{*}=z \sum_{j} w_{j} e e_{A} w_{j}^{*} \\
& =z g .
\end{aligned}
$$

Since $B_{1}=C^{*}\left\langle C^{*}\left\langle A, e_{P}\right\rangle, e_{A}\right\rangle, g \in B_{1}^{\prime} \cap B_{1}^{\infty}$.

To prove that the double dual conditional expectation $\hat{\hat{E}}$ has the Rokhlin property, we will show that $g$ is the Rokhlin projection of $\hat{\hat{E}}$. Since $e z e=\hat{E}(z) e$ for any $z \epsilon$ $C^{*}\left\langle A, w_{P}\right\rangle$, by Remark 17(5), there exists an isomorphism $\pi$ : $C^{*}\left\langle C^{*}\left\langle A, e_{P}\right\rangle, e_{A}\right\rangle \rightarrow C^{*}\left\langle C^{*}\left\langle A, e_{P}\right\rangle, e\right\rangle$ such that $\pi\left(e_{A}\right)=e$ and $\pi(z)=z$ for $z \in C^{*}\left\langle A, e_{P}\right\rangle$. Then

$$
\begin{aligned}
\hat{\hat{E}}^{\infty}(g) & =\sum_{i} w_{i} e \hat{\hat{E}}^{\infty}\left(e_{A}\right) w_{i}^{*} \\
& =\sum_{i} w_{i}(\operatorname{Index} E)^{-1} e w_{i}^{*} \\
& =(\operatorname{Index} E)^{-1} \sum_{i} w_{i} \pi\left(e_{A}\right) w_{i}^{*} \\
& =(\operatorname{Index} E)^{-1} \pi\left(\sum_{i} w_{i} e_{A} w_{i}^{*}\right) \\
& =(\operatorname{Index} E)^{-1} 1 \\
& =(\operatorname{Index} \hat{\hat{E}})^{-1} 1,
\end{aligned}
$$

hence $\hat{\hat{E}}$ has the Rokhlin property.

\subsection{Main Results}

Theorem 23. Let $1 \in P \subset A$ be an inclusion of unital $C^{*}$ algebras with a finite Watatani index and $E: A \rightarrow P$ a faithful conditional expectation. Suppose that A has the LP property and $E$ has the Rokhlin property. Then $P$ has the LP property. 
Proof. Let $x \in P$ and $\varepsilon>0$. Since $A$ has the LP property, $x$ can be approximated by a line sum of projection $\sum_{i=1}^{n} \lambda_{i} p_{i}\left(p_{i} \in\right.$ $A)$ such that $\left\|x-\sum_{i=1}^{n} \lambda_{i} p_{i}\right\|<\varepsilon$.

Since $\beta: A^{\infty} \rightarrow P^{\infty}$ is an injective $*$-homomorphism by Proposition 21, we have

$$
\left\|\beta\left(x-\sum_{i=1}^{n} \lambda_{i} p\right)\right\|=\left\|\beta(x)-\sum_{i=1}^{n} \lambda_{i} \beta\left(p_{i}\right)\right\|<\varepsilon .
$$

Since $\beta_{\mid P}=i d$, we have $\left\|x-\sum_{i=1}^{n} \lambda_{i} \beta\left(p_{i}\right)\right\|<\varepsilon$. Each projection in $P^{\infty}$ can be lifted to a projection in $\ell^{\infty}(\mathbb{N}, P)$, so we can find a set of projections $\left\{q_{i}\right\}_{i=1}^{n} \subset P$ such that

$$
\left\|x-\sum_{i=1}^{n} \lambda_{i} q_{i}\right\|<\varepsilon
$$

Therefore, $P$ has the LP property.

Theorem 24. Let $\alpha$ be an action of a finite group $G$ on a simple unital $C^{*}$-algebra $A$ and $E$ be canonical conditional expectation from $A$ to the fixed point algebra $P=A^{\alpha}$ defined by

$$
E(x)=\frac{1}{\# G} \sum_{g \in G} \alpha_{g}(x), \quad \text { for } x \in A,
$$

where $\# G$ is the order of $G$. Suppose that $\alpha$ has the Rokhlin property. We have, then, that if $A$ has the LP property, the fixed point algebra and the crossed product $A \rtimes_{\alpha} G$ have the LP property.

Before giving the proof, we need the following two lemmas, which must be well known.

Lemma 25. Under the same conditions in Theorem 24 consider the following two basic constructions:

$$
\begin{gathered}
A^{\alpha} \subset A \subset C^{*}\left\langle A, e_{P}\right\rangle \subset C^{*}\left\langle B, e_{A}\right\rangle \quad\left(B=C^{*}\left\langle A, e_{P}\right\rangle\right) \\
\left(A^{\alpha}\right) \subset A \subset A \rtimes_{\alpha} G \subset C^{*}\left\langle A \rtimes_{\alpha} G, e_{F}\right\rangle,
\end{gathered}
$$

where $F: A \rtimes_{\alpha} G \rightarrow A$ is a canonical conditional expectation. Then there is an isomorphism $\pi: C^{*}\left\langle A, e_{P}\right\rangle \rightarrow A \rtimes_{\alpha} G$ and $\tilde{\pi}: C^{*}\left\langle B, e_{A}\right\rangle \rightarrow C^{*}\left\langle A \rtimes_{\alpha} G, e_{F}\right\rangle$ such that

(1) $\pi(a)=a$ for all $a \in A$,

(2) $\pi\left(e_{p}\right)=q$, where $q=(1 /|G|) \sum_{g \in G} u_{g}$,

(3) $A \rtimes_{\alpha} G=C^{*}\langle A, q\rangle$,

(4) $\tilde{\pi}(b)=\pi(b)$ for all $b \in B$,

(5) $\tilde{\pi}\left(e_{A}\right)=e_{F}$.

Moreover, we have

(6) $F \circ \pi=\hat{E}$ and $\pi \circ \hat{\hat{E}}=\hat{F} \circ \tilde{\pi}$.
Proof. At first we prove condition (3). Since $\alpha$ is outer, $\alpha$ is saturated by [21, Proposition 4.9]; that is,

$$
\begin{aligned}
& A \rtimes_{\alpha} G \\
& =\frac{\text { linear span of }\left\{\sum_{g \in G}\left(\alpha_{g}(x) u_{g}\right)^{*}\left(\sum_{g \in G} \alpha_{g}(y) u_{g}\right) \mid x, y \in A\right\}}{=} \\
& =\text { linear span of }\left\{\frac{1}{|G|} \sum_{g \in G} x^{*} \alpha_{g}(y) u_{g} \mid x, y \in A\right\} .
\end{aligned}
$$

On the contrary, for any $x, y \in A$, we have

$$
\begin{aligned}
x q y & =x \frac{1}{|G|} \sum_{g \in G} u_{g} y \\
& =\frac{1}{|G|} x \sum_{g \in G} u_{g} y u_{g}^{*} u_{g} \\
& =\frac{1}{|G|} \sum_{g \in G} x \alpha_{g}(y) u_{g},
\end{aligned}
$$

hence $A \rtimes_{\alpha} G=C^{*}\langle A, q\rangle$.

Since for any $a \in A$

$$
\begin{aligned}
q a q & =\frac{1}{|G|^{2}} \sum_{g \in G} u_{g} a \sum_{h \in G} u_{h} \\
& =\frac{1}{|G|^{2}} \sum_{g, h \in G} u_{g} a u_{g}^{*} u_{g} h \\
& =\frac{1}{|G|^{2}} \sum_{g, h \in G} \alpha_{g}(a) u_{g h} \\
& =\frac{1}{|G|} \sum_{g \in G} \alpha_{g}(a) \frac{1}{|G|} \sum_{h \in G} u_{g h} \\
& =E(a) q,
\end{aligned}
$$

by Remark 17(5) there is an isomorphism $\pi: C^{*}\left\langle A, e_{P}\right\rangle \rightarrow$ $C^{*}\langle A, q\rangle=A \rtimes_{\alpha} G$ such that $\pi(a)=a$ for any $a \in A$ and $\pi\left(e_{P}\right)=q$. Hence conditions (1) and (2) are proved.

By the similar steps we will show conditions (4) and (5). Since for any $x, y, a, b \in A$

$$
\begin{aligned}
\left(e_{F} \pi\left(x e_{P} y\right) e_{F}\right)(a q b) & =\left(e_{F} x q y\right) F\left(\frac{1}{|G|} \sum_{g \in G} a \alpha_{g}(b) u_{g}\right) \\
& =\frac{1}{|G|}\left(e_{F} x q y a b\right) \\
& =\frac{1}{|G|^{2}} x y a b .
\end{aligned}
$$


On the contrary,

$$
\begin{aligned}
\pi\left(\hat{E}\left(x e_{P} y\right)\right) e_{F}(a q b) & =\pi\left(\frac{1}{|G|} x y\right) \frac{1}{|G|} a b \\
& =\frac{1}{|G|^{2}} x y a b .
\end{aligned}
$$

Hence, we have $e_{F} \pi\left(x e_{p} y\right) e_{F}=\pi\left(\hat{E}\left(x e_{P} y\right)\right)$. By Remark 17(5), there is an isomorphism $\tilde{\pi}: C^{*}\left\langle B, e_{A}\right\rangle \rightarrow C^{*}\left\langle A \rtimes_{\alpha} G, e_{F}\right\rangle$ such that $\tilde{\pi}(b)=\pi(b)$ for any $b \in B$ and $\tilde{\pi}\left(e_{A}\right)=e_{F}$.

The condition (6) comes from the direct computation.

Lemma 26. Under the same conditions in Lemma $25 C^{*}\left\langle A \rtimes_{\alpha} G, e_{F}\right\rangle$ is isomorphic to $M_{|G|}(A)$.

Proof. Note that $\left\{\left(u_{g}^{*}, u_{g}\right)\right\}_{g \in G}$ is a quasi-basis for $F$. By [17, Lemma 3.3.4], there is an isomorphism from $C^{*}\left\langle A \rtimes_{\alpha} G, e_{F}\right\rangle$ to $r M_{|G|}(A) r$, where $r=\left[E\left(u_{g}^{*} u_{h}\right)\right]_{g, h \in G}=I_{|G|}$. Hence $C^{*}\left\langle A \rtimes_{\alpha} G, e_{F}\right\rangle$ is isomorphic to $M_{|G|}(A)$.

Proof of Theorem 24. Let $\left\{e_{g}\right\}_{g \in G}$ be the Rokhlin projection of $E$. From Proposition 20, $E: A \rightarrow A^{G}$ is of index finite and has a projection $e \in A^{\prime} \cap A^{\infty}$ such that $E^{\infty}(e)=(1 /|G|) 1$. Note that index $E=|G|$ and $e=e_{1}$. Consider the basic construction

$$
A^{G} \subset A \subset C^{*}\left\langle A, e_{P}\right\rangle \subset C^{*}\left\langle B, e_{A}\right\rangle \quad\left(B=C^{*}\left\langle A, e_{P}\right\rangle\right) .
$$

Since $A$ is simple, the map $A \ni x \mapsto x e$ is injective, hence we know that $E$ has the Rokhlin property. Therefore, $A^{G}$ has the LP property by Theorem 23.

Since $C^{*}\left\langle A \rtimes_{\alpha} G, e_{F}\right\rangle$ is isomorphic to $M_{|G|}(A)$ by Lemma 26 and $A$ has the LP property, $C^{*}\left\langle A \rtimes_{\alpha} G, e_{F}\right\rangle$ has the LP property. Hence, $C^{*}\left\langle B, e_{A}\right\rangle$ has the LP property, because that $C^{*}\left\langle A \rtimes_{\alpha} G, e_{F}\right\rangle$ is isomorphic to $C^{*}\left\langle B, e_{A}\right\rangle$ from Lemma 25. From Proposition 22, $\hat{\hat{E}}: C^{*}\left\langle B, e_{A}\right\rangle \rightarrow$ $C^{*}\left\langle A, e_{P}\right\rangle$ has the Rokhlin property, hence we conclude that $C^{*}\left\langle A, e_{P}\right\rangle$ has the LP property by Theorem 23 . Since $C^{*}\left\langle A, e_{P}\right\rangle$ is isomorphic to $A \rtimes_{\alpha} G$ by Lemma 25 , we conclude that $A \rtimes_{\alpha} G$ has the LP property.

\section{Remark 27.}

(1) When an action $\alpha$ of a finite group $G$ does not have the Rokhlin property, we have an example of simple unital $C^{*}$-algebra with the LP property such that the fixed point algebra $A^{G}$ does not have the LP property by Example 14. Note that the action $\alpha$ does not have the Rokhlin property.

(2) When an action of a finite group $G$ on a unital $C^{*}$ algebra $A$ has the Rokhlin property, the crossed product can be locally approximated by the class of matrix algebras over corners of $A$ [22, Theorem 3.2]. Many kinds of properties are preserved by this method such as AF algebras [23], AI algebras, AT algebras, simple AH algebras with slow dimension growth and real rank zero [22], D-absorbing separable unital $C^{*}$ algebras for a strongly self-absorbing $C^{*}$-algebras D [24], simple unital separable strongly self-absorbing $C^{*}$-algebras [20], and unital Kirchberg $C^{*}$-algebras [22]. Like the ideal property [25], however, since the LP property is not preserved by passing to corners by Lemma 12, we cannot apply this method to determine the LP property of the crossed products.

We could also have many examples which shows that the LP property is preserved under the formulation of crossed products from the following observation.

Let $A$ be an infinite dimensional simple $C^{*}$-algebra and let $\alpha$ be an action from a finite group $G$ on $\operatorname{Aut}(A)$. Recall that $\alpha$ has the tracial Rokhlin property if for every finite set $F \subset A$, every $\varepsilon>0$, and every positive element $x \in A$ with $\|x\|=1$, there are mutually orthogonal projections $e_{g} \in A$ for $g \in G$ such that

(1) $\left\|\alpha_{g}\left(e_{h}\right)-e_{g h}\right\|<\varepsilon$ for all $g, h \in G$ and all $a \in F$,

(2) $\left\|e_{g} a-a e_{g}\right\|<\varepsilon$ for all $g \in G$ and all $a \in F$,

(3) with $e=\sum_{g \in G} e_{g}$, the projection $1-e$ is the Murray-von Neumann equivalent to a projection in the hereditary subalgebra of $A$ generated by $x$,

(4) with $e$ as in (3), we have $\|$ exe $\|>1-\varepsilon$.

It is obvious that the tracial Rokhlin property is weaker than the Rokhlin property.

Proposition 28. Let $\alpha$ be an action of a finite group $G$ on a simple unital $C^{*}$-algebra $A$ with a unique tracial state. Suppose that $\alpha$ has the tracial Rokhlin property. If $A$ has the LP property, then the crossed product $A \rtimes_{\alpha} G$ has the LP property.

Proof. From [26, Proposition 5.7], the restriction map from tracial states on the crossed product $A \rtimes_{\alpha} G$ to $\alpha$-invariant tracial states on $A$ is isomorphism. Hence, $A \rtimes_{\alpha} G$ has a unique tracial state.

Since $\alpha$ is a pointwise outer (i.e., for any $g \in G \backslash\{0\} \alpha_{g}$ is outer) by [23, Lemma 1.5], $A \rtimes_{\alpha} G$ is simple.

Therefore, by [1, Corollary 4], $A \rtimes_{\alpha} G$ has the LP property.

Remark 29. There are many examples of actions $\alpha$ of finite groups on simple unital $C^{*}$-algebras with real rank zero and a unique tracial state such that $\alpha$ has the tracial Rokhlin property, see $[23,26]$.

\section{Acknowledgment}

The research of H. Osaka was partially supported by the JSPS grant for Scientific Research no. 23540256. 


\section{References}

[1] G. K. Pedersen, "The linear span of projections in simple $C^{*}$ algebras," Journal of Operator Theory, vol. 4, no. 2, pp. 289-296, 1980.

[2] B. Blackadar, O. Bratteli, G. A. Elliott, and A. Kumjian, "Reduction of real rank in inductive limits of $C^{*}$-algebras," Mathematische Annalen, vol. 292, no. 1, pp. 111-126, 1992.

[3] O. Bratteli and G. A. Elliott, "Small eigenvalue variation and real rank zero," Pacific Journal of Mathematics, vol. 175, no. 1, pp. 4759, 1996.

[4] G. A. Elliott, T. M. Ho, and A. S. Toms, "A class of simple $C^{*}$ algebras with stable rank one," Journal of Functional Analysis, vol. 256, no. 2, pp. 307-322, 2009.

[5] T. M. Ho, "On the property SP of certain AH algebras," Comptes Rendus Mathématiques de l'Académie des Sciences. La Société Royale du Canada, vol. 29, no. 3, pp. 81-86, 2007.

[6] K. R. Goodearl, "Notes on a class of simple $C^{*}$-algebras with real rank zero," Publicacions Matemàtiques, vol. 36, no. 2, pp. 637-654, 1992.

[7] A. S. Toms and W. Winter, "The Elliott conjecture for Villadsen algebras of the first type," Journal of Functional Analysis, vol. 256, no. 5, pp. 1311-1340, 2009.

[8] A. S. Toms, "On the classification problem for nuclear $C^{*}$ algebras," Annals of Mathematics. Second Series, vol. 167, no. 3, pp. 1029-1044, 2008.

[9] G. A. Elliott, "On the classification of inductive limits of sequences of semisimple finite-dimensional algebras," Journal of Algebra, vol. 38, no. 1, pp. 29-44, 1976.

[10] G. A. Elliott and G. Gong, "On the classification of $C^{*}$-algebras of real rank zero. II," Annals of Mathematics. Second Series, vol. 144, no. 3, pp. 497-610, 1996.

[11] G. A. Elliott, G. Gong, and L. Li, "On the classification of simple inductive limit $C^{*}$-algebras. II. The isomorphism theorem," Inventiones Mathematicae, vol. 168, no. 2, pp. 249-320, 2007.

[12] B. Blackadar, M. Dădărlat, and M. Rørdam, "The real rank of inductive limit $C^{*}$-algebras," Mathematica Scandinavica, vol. 69, no. 2, pp. 267-276, 1991.

[13] R. V. Kadison, "Diagonalizing matrices," American Journal of Mathematics, vol. 106, no. 6, pp. 1451-1468, 1984.

[14] L. G. Brown and G. K. Pedersen, " $C^{*}$-algebras of real rank zero," Journal of Functional Analysis, vol. 99, no. 1, pp. 131-149, 1991.

[15] G. A. Elliott, "A classification of certain simple $C^{*}$-algebras," in Quantum and Non-Commutative Analysis (Kyoto, 1992), vol. 16 of Mathematical Physics Studies, pp. 373-385, Kluwer Academic Publishers, Dodrecht, The Netherlands, 1993.

[16] K. Thomsen, "Inductive limits of interval algebras: the tracial state space," American Journal of Mathematics, vol. 116, no. 3, pp. 605-620, 1994.

[17] Y. Watatani, Index for $\mathrm{C}^{*}$-Subalgebras, Memoirs of the American Mathematical Society, American Mathematical Society, Providence, RI, USA, 1990.

[18] M. Izumi, "Finite group actions on $C^{*}$-algebras with the Rohlin property. I," Duke Mathematical Journal, vol. 122, no. 2, pp. 233280, 2004.

[19] K. Kodaka, H. Osaka, and T. Teruya, "The Rohlin property for inclusions of $C^{*}$-algebras with a finite Watatani index," Contemporary Mathematics, vol. 503, pp. 177-195, 2009.

[20] H. Osaka and T. Teruya, "Strongly self-absorbing property for inclusions of $C^{*}$-algebras with a finite Watatani index," Transactions of the American Mathematical Society. In press, http://arxiv.org/abs/1002.4233.
[21] J. A. Jeong and G. H. Park, "Saturated actions by finitedimensional Hopf ${ }^{*}$-algebras on $C^{*}$-algebras," International Journal of Mathematics, vol. 19, no. 2, pp. 125-144, 2008.

[22] H. Osaka and N. C. Phillips, "Crossed products by finite group actions with the Rokhlin property," Mathematische Zeitschrift, vol. 270, no. 1-2, pp. 19-42, 2012.

[23] N. C. Phillips, "The tracial Rokhlin property for actions of finite groups on $C^{*}$-algebras," American Journal of Mathematics, vol. 133, no. 3, pp. 581-636, 2011.

[24] I. Hirshberg and W. Winter, "Rokhlin actions and self-absorbing $C^{*}$-algebras," Pacific Journal of Mathematics, vol. 233, no. 1, pp. 125-143, 2007.

[25] C. Pasnicu and C. N. Phillips, "Permanence properties for crossed products and fixed point algebras of finite groups," http://arxiv.org/abs/1208.3810.

[26] S. Echterhoff, W. Lück, N. C. Phillips, and S. Walters, "The structure of crossed products of irrational rotation algebras by finite subgroups of $S L_{2}(Z)$," Journal für die Reine und Angewandte Mathematik, vol. 639, pp. 173-221, 2010. 


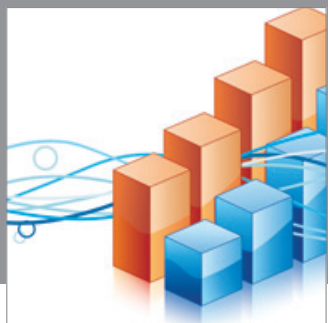

Advances in

Operations Research

mansans

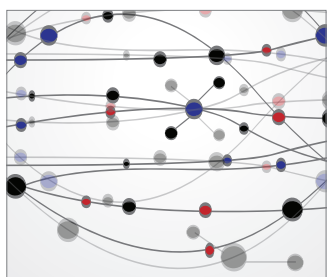

The Scientific World Journal
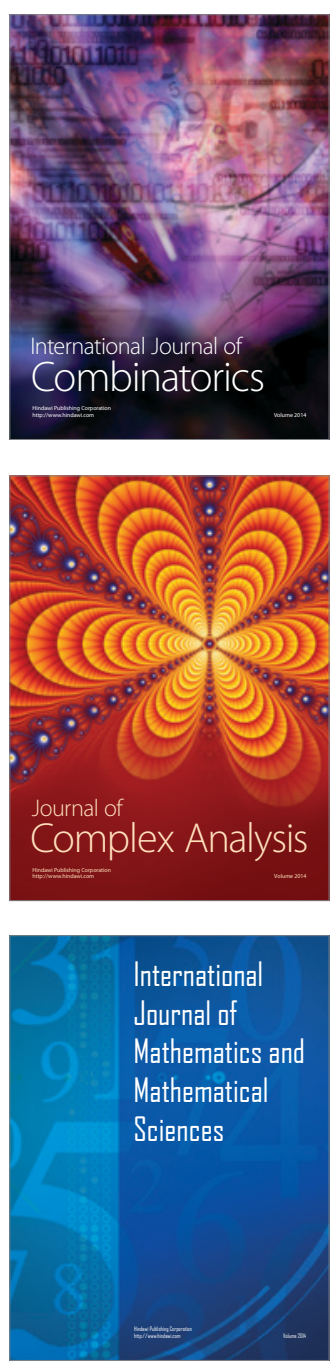
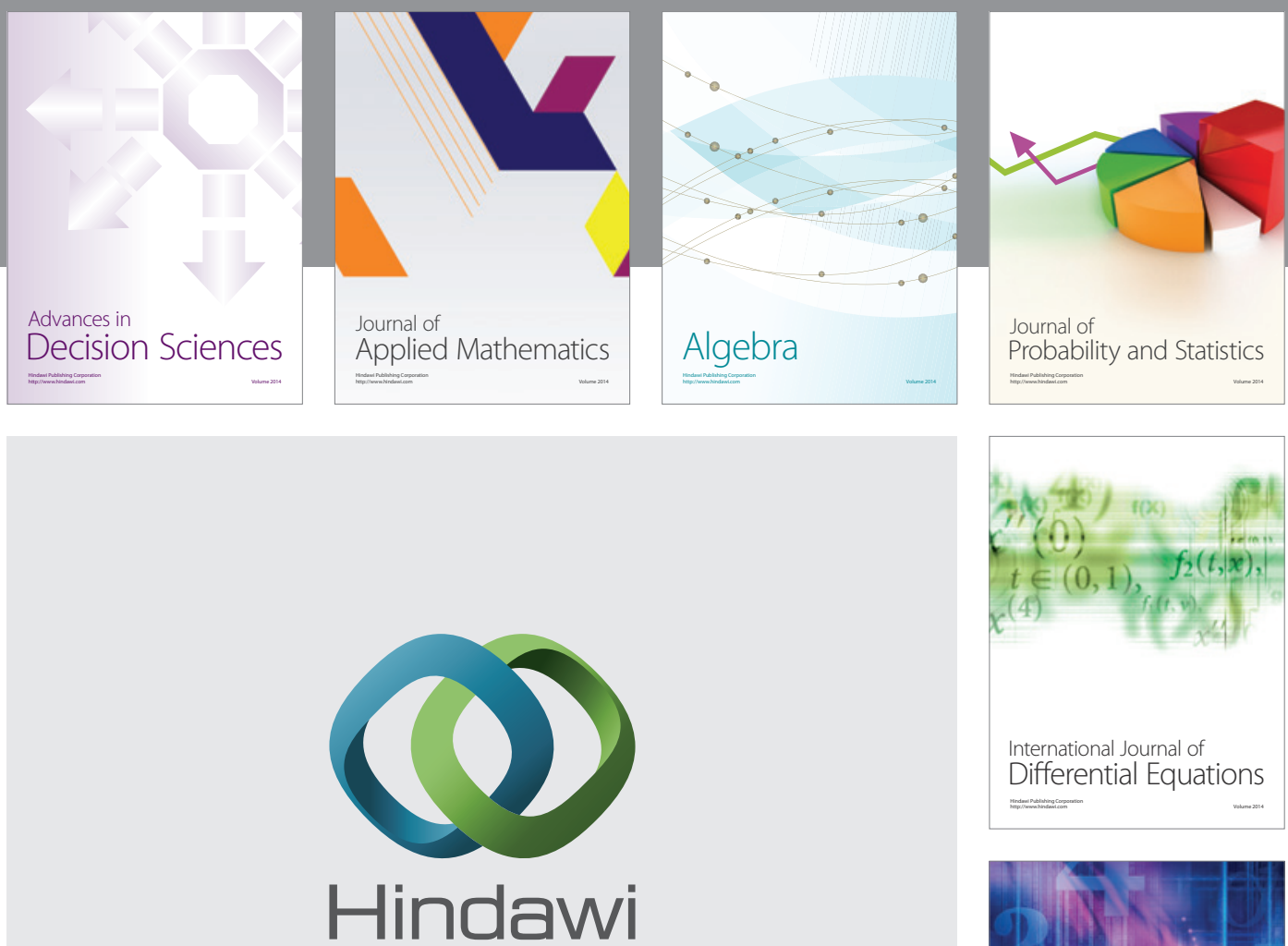

Submit your manuscripts at http://www.hindawi.com
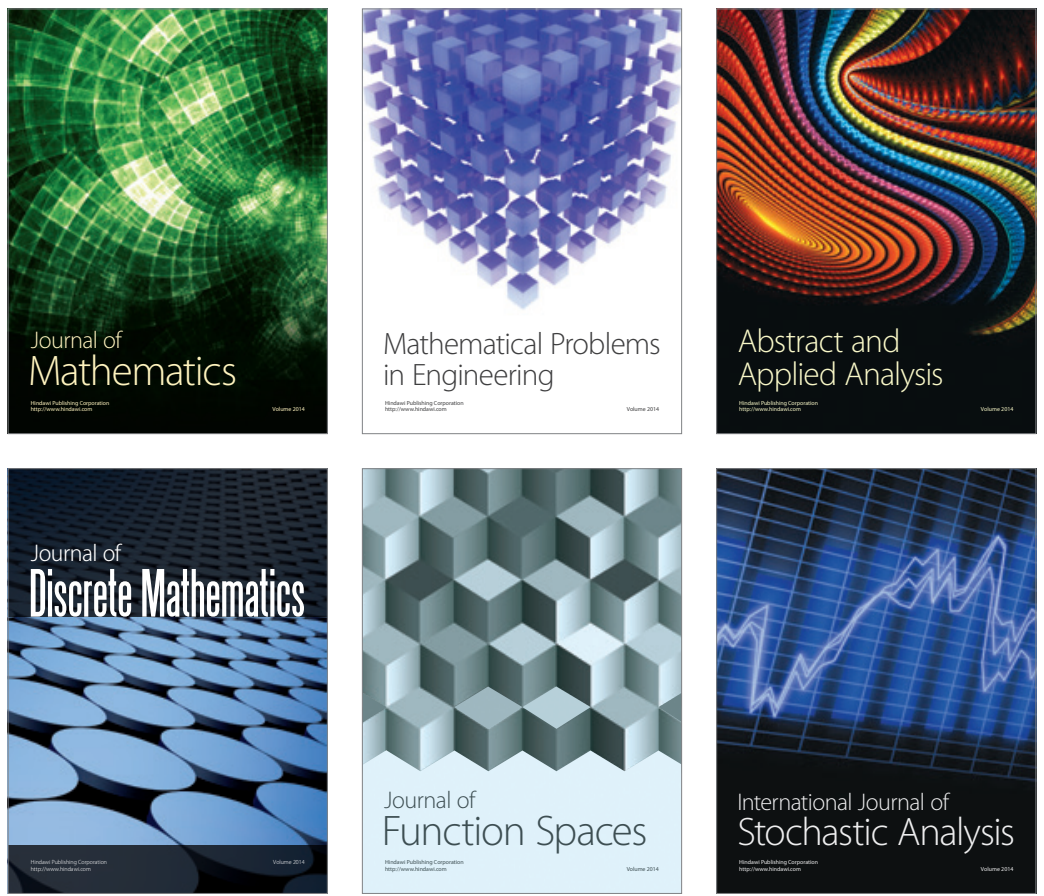

Journal of

Function Spaces

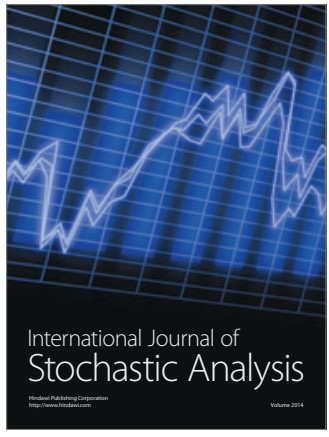

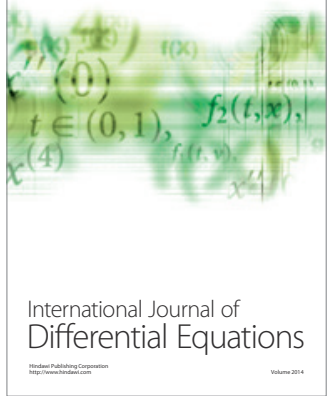
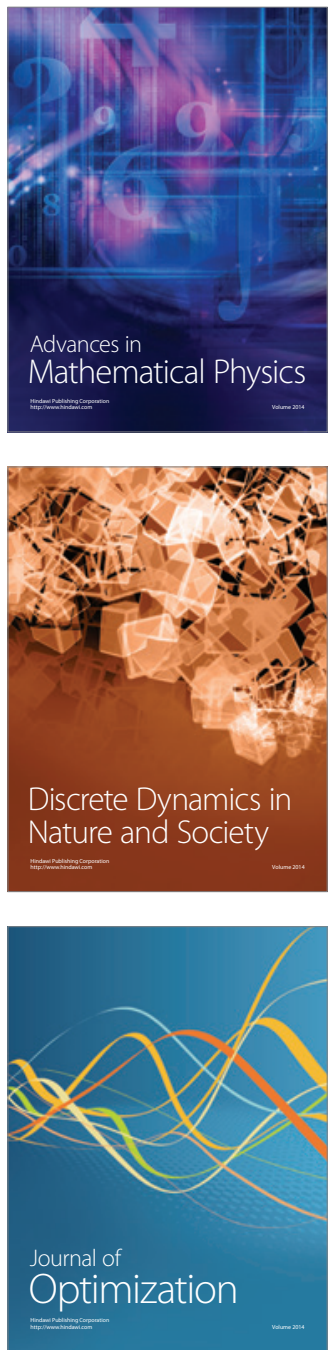